\title{
Neurocognitive Dysfunction According to Hypoperfusion Territory in Patients With Moyamoya Disease
}

\author{
Chang Gu Kang, MD, Min Ho Chun, MD, PhD, Jung-A Kang, MD, Kyung Hee Do, MD, Su Jin Choi, MD \\ Department of Rehabilitation Medicine, Asan Medical Center, University of Ulsan College of Medicine, Seoul, Korea
}

\begin{abstract}
Objective To demonstrate the prevalence of cerebral hypoperfusion without focal cerebral lesions in patients with Moyamoya disease (MMD), and the relationship between areas of hypoperfusion and cognitive impairment.

Methods Twenty-six MMD patients were included. Patients were categorized according to the presence/absence of hypoperfusion in the frontal, parietal, temporal, and occipital lobes on brain single-photon-emission computed tomography (SPECT) after acetazolamide challenge. Computerized neuropsychological test (CNT) results were compared between groups.

Results Only 3 patients showed normal cerebral perfusion. Baseline characteristics were similar between groups. Patients with frontal lobe hypoperfusion showed lower scores in visual continuous performance test (CPT), auditory CPT, forward digit span test, backward digit span test, verbal learning test, and trail-making test. Patients with parietal lobe hypoperfusion showed lower backward digit span test, visual learning test, and trail-making test scores. Related to temporal and occipital lobes, there were no significant differences in CNT results between the hypoperfusion and normal groups.

Conclusion MMD patients without focal cerebral lesion frequently exhibit cerebral hypoperfusion. MMD patients with frontal and parietal hypoperfusion had abnormal CNT profiles, similar to those with frontal and parietal lesions. It is suggested that the hypoperfusion territory on brain SPECT without focal lesion may affect the characteristics of neurocognitive dysfunction in MMD patients.
\end{abstract}

Keywords Moyamoya disease, Cerebral hypoperfusion, Cognition

\section{INTRODUCTION}

Moyamoya disease (MMD) is a rare cause of stroke in children and young adults, characterized by an occlusive arteriopathy involving the intracranial segments of the internal carotid and proximal anterior cerebral arteries

Received June 10, 2016; Accepted August 23, 2016

Corresponding author: Min Ho Chun

Department of Rehabilitation Medicine, Asan Medical Center, University of Ulsan College of Medicine, 88 Olympic-ro 43-gil, Songpa-gu, Seoul 05505, Korea. Tel: +82-2-3010-3800, Fax: +82-2-3010-6964, E-mail: mhchun@amc.seoul.kr

ORCID: Chang Gu Kang (http://orcid.org/0000-0002-2999-9474); Min Ho Chun (http://orcid.org/0000-0001-8666-7225); Jung-A Kang (http://orcid. org/0000-0001-5275-278X); Kyung Hee Do (http://orcid.org/0000-0003-4235-8759); Su Jin Choi (http://orcid.org/0000-0002-3443-2237).

(c) This is an open-access article distributed under the terms of the Creative Commons Attribution Non-Commercial License (http://creativecommons.org/ licenses/by-nc/4.0) which permits unrestricted noncommercial use, distribution, and reproduction in any medium, provided the original work is properly cited. Copyright ( 2017 by Korean Academy of Rehabilitation Medicine 
[1]. Its characteristic appearance on cerebral angiogram resembles a 'puff of smoke', which was first described by Takeuchi and Shimizu [2] in 1957. The most common presentation is recurrent transient ischemic attacks or strokes in childhood, resulting in significant morbidity [3]. Ischemic symptoms are usually caused by hemodynamically mediated perfusion failure, rather than thromboembolism [4]. Patients with large artery thrombosis or hypoxic cerebral injury show cognitive impairment due to the cerebral hypoperfusion $[5,6]$. MMD patients suffer from chronic hypoperfusion and hemodynamic compromise [7]; therefore, they might have neurocognitive dysfunction, which affects the daily activities and functional outcomes. Therefore, early detection of neurocognitive dysfunction and prompt rehabilitation are important.

Various methods, such as positron emission tomography and xenon computed tomography, have been used to study cerebral blood flow in patients with MMD. Singlephoton emission computed tomography (SPECT) is a relatively new technique that is being increasingly used for evaluating cerebral hypoperfusion. It detects the early foci of cerebral hypoperfusion, and may help guide the use of reperfusion therapies. Brain perfusion SPECT may be used to evaluate regional cerebral blood flow in MMD, and acetazolamide challenge can reveal the cerebral blood flow reserve [8].

In a previous study, 30 children having MMD without focal cerebral lesions showed lower Wechsler Intelligence Scale scores [9]. Among adult patients having MMD without focal cerebral lesions, a large proportion of patients showed cognitive impairment on the Wechsler Adult Intelligence Test-III, as well as impaired processing speed, verbal memory, verbal fluency, and executive function [10]. As impaired cognitive functions were mediated by subcortical and frontal regions, which are the usual areas of hypoperfusion in patients with MMD, previous results suggested small vessel disease caused by chronic hypoperfusion as the mechanism of cognitive impairment $[10,11]$. However, no studies have evaluated the correlation between areas of cerebral hypoperfusion and neurocognitive impairment.

The aims of this study were to evaluate the prevalence of cerebral hypoperfusion in MMD, and the relationship between areas of hypoperfusion and cognitive impairment in MMD patients without focal cerebral lesions. We attempted to investigate the neurocognitive profiles of adults with MMD according to territory of cerebral hypoperfusion on brain SPECT.

\section{MATERIALS AND METHODS}

From January 2001 to May 2013, 26 patients referred to the Department of Rehabilitation, Asan Medical Center, were recruited. Following inclusion criteria were fulfilled: (1) age 18-60 years, (2) diagnosis of MMD by angiographic evidence of Moyamoya collateral vessels and stenosis, (3) absence of parenchymal stroke lesion, and (4) absence of history of developmental disorder or any neurological condition known to cause cognitive impairment. The study was approved by Asan Medical Center Institutional Review Board and the local Ethics Committee (IRB No. S-2013-1201-0007).

\section{Measures}

Baseline characteristics, such as age, gender, symptom duration, number of events, lesion on angiography, and SPECT findings, were assessed through the medical record review. The Korean version of Modified Barthel Index (K-MBI) was used to assess the daily activity performance [12]. The brain SPECT were reviewed by nuclear medicine specialists. Subjects were divided according to the presence/absence of hypoperfusion in each lobe, after acetazolamide challenge (Fig. 1). Next, the characteristics of computerized neuropsychological test (CNT; MaxMedica, Seoul, Korea) [13] of the hypoperfusion group and the normal perfusion group were compared for each lobe.

CNT included visual continuous performance test (CPT), auditory CPT, forward digit span test, backward digit span test, forward visual span test, backward visual span test, verbal learning test, visual learning test, and trail making test-A. Visual CPT records the response time of the patient pushing the button on seeing number 3 on the computer screen. Auditory CPT records the response time of the patient pushing a button whenever he or she hears the number 3 . In the verbal learning test, 15 target words were repeated 5 times on a speaker. After 20 minutes, we scored the number of words they recalled initially, and the recall after presenting on the screen a list of 30 words containing the previous 15 words. For the spatial memory test, forward visual span test, backward visual span test, and visual learning test were performed. For 


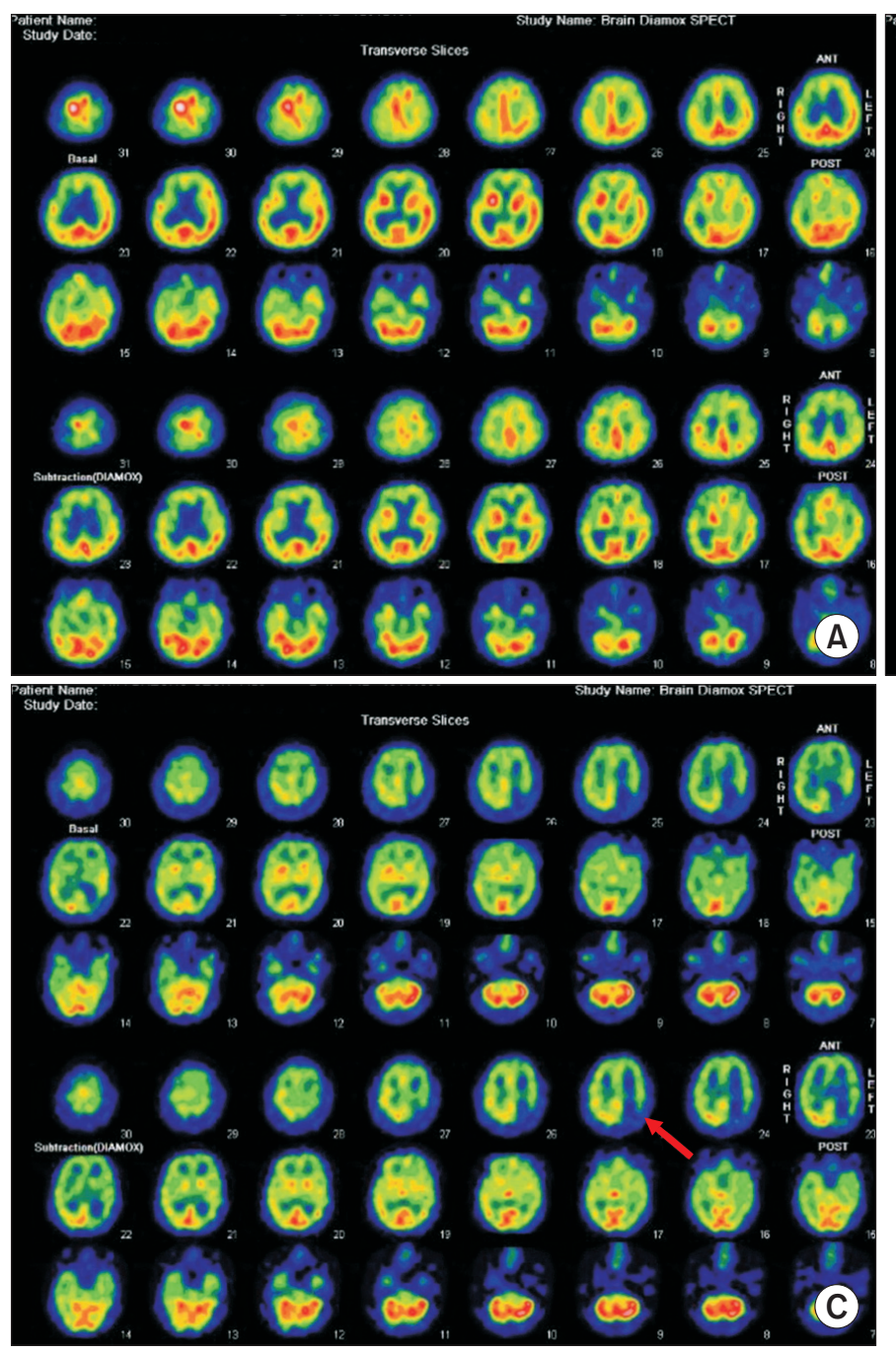

the visual learning test, 15 different figures were initially presented sequentially; following this, 30 figures, including the 15 figures that were previously presented, were shown. Similar to the auditory CPT, the 15 figures were repeatedly shown 5 times. Recalls were tested 20 minutes later, and after showing all 30 figures on the screen. For the visual motor coordination test, we evaluated the time taken to draw lines connecting 25 small circles on the screen, containing numbers $1-25$, in each small circle (trail-making test-type A) [13].

\section{Statistical analysis}

SPSS software ver. 18.0.0 (SPSS Inc., Chicago, IL, USA) was used for statistical analysis, and the p-value significance was set at 0.05 . Baseline characteristics were summarized with descriptive statistics, including mean, standard deviation, and $95 \%$ confidence interval) for

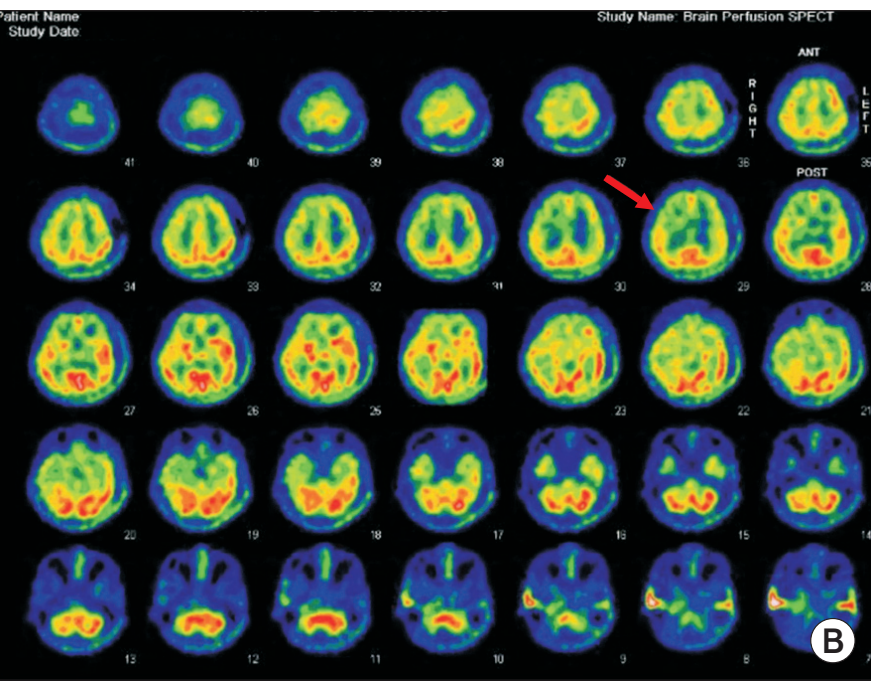

Fig. 1. Classification of hypoperfusion in each lobe, relative to the cerebellar signal activity on SPECT. (A) SPECT image is normal. (B) SPECT image shows right frontallobe hypoperfusion (red arrow). (C) SPECT image shows left parietal lobe hypoperfusion (red arrow). SPECT, single-photon emission computed tomography.

continuous variables, and frequencies for categorical variables. Fisher exact test was computed for comparisons of categorical variables (gender, primary event, and coexisting hypoperfusion), and Mann-Whitney test were used for comparisons of continuous variables (age, symptom duration, and K-MBI). To compare CNT scores between groups, Mann-Whitney test was used.

\section{RESULTS}

The baseline characteristics are presented in Table 1 . Of the 26 patients, 15 had frontal lobe hypoperfusion (FLH), 10 had parietal lobe hypoperfusion (PLH), 8 had temporal lobe hypoperfusion, and 7 had occipital-lobe hypoperfusion on brain SPECT. Only 3 of the 26 patients (11.5\%) had no hypoperfusion, and they showed normal neurocognitive profiles (MMSE 28.6 \pm 0.6 ; CNT result was 
normal).

Focusing on the frontal lobe, the mean age of the FLH group was 42.1 years and that of the frontal-lobe-normal

Table 1. Baseline characteristics of patients with Moyamoya disease

\begin{tabular}{lc}
\hline \multicolumn{1}{c}{ Characteristic } & Value \\
\hline Age (yr) & $41.8 \pm 12.9$ \\
\hline Gender (male:female) & $7: 19$ \\
\hline Age of onset (yr) & $40.0 \pm 12.9$ \\
\hline Symptom duration (mo) & $11.2 \pm 21.4$ \\
\hline Primary event & \\
\hline TIA & $9(34.6)$ \\
\hline Headache & $3(11.5)$ \\
\hline None & $14(53.8)$ \\
\hline K-MBI & $69.2 \pm 15.7$ \\
\hline K-MMSE & $22.0 \pm 4.7$ \\
\hline
\end{tabular}

Values are presented as mean \pm standard deviation or number (\%).

TIA, transient ischemic attack; SPECT, single-photon emission computed tomography; K-MBI, Korean version of the Modified Barthel Index; K-MMSE, Korean version of the Mini-Mental Status Examination.
(FLN) group was 44.1 years $(\mathrm{p}=0.45)$. The age of MMD onset was 39.5 years in the FLH group, and 40.7 years in the FLN group ( $\mathrm{p}=0.81$ ). The primary event (transient ischemic attack, or headache or none), coexisting hypoperfusion on SPECT (none, parietal, parieto-temporal, parieto-occipital, temporo-occipital, temporal), and KMBI score were not significantly different between the FLH and the FLN groups (Table 2). The K-MMSE score had a tendency to be lower in the FLH group (20.5) than in the FLN group (24.0), but this was not statistically significant $(\mathrm{p}=0.054)$. Compared with the FLN group, brain SPECT of the FLH group showed lower scores of visual CPT, auditory CPT, forward digit span test, backward digit span test, verbal learning test, and trail-making test $(\mathrm{p}<0.05)$ (Table 3).

Focusing on the parietal lobe, the mean age of the PLH group was 39.3 years and that of the parietal-lobe-normal (PLN) group was 42.5 years $(\mathrm{p}=0.32)$. The age of MMD onset was 38.5 years in the PLH group, and 39.9 years in the PLN group ( $\mathrm{p}=0.74)$. The primary event, coexisting hypoperfusion on SPECT, and K-MBI score were not significantly different between the groups (Table 4). The KMMSE score had a tendency to be lower in the PLH group

Table 2. Baseline characteristics of Moyamoya disease patients on frontal-lobe SPECT

\begin{tabular}{|lccc}
\hline & $\begin{array}{c}\text { With hypoperfusion } \\
\text { of the frontal lobe (n=15) }\end{array}$ & $\begin{array}{c}\text { Without hypoperfusion } \\
\text { of the frontal lobe (n=11) }\end{array}$ & p-value \\
\hline Age (yr) & $42.1 \pm 13.1$ & $44.1 \pm 12.8$ & 0.45 \\
\hline Onset age (yr) & $39.5 \pm 12.6$ & $40.7 \pm 13.9$ & 0.81 \\
\hline Gender (male:female) & $3: 12$ & $4: 7$ & 0.41 \\
\hline Symptom duration (mo) & $29.1 \pm 14.1$ & $39.1 \pm 50.2$ & 0.43 \\
\hline Primary event & & & 0.74 \\
\hline TIA & $5(33.3)$ & $4(36.3)$ & \\
\hline Headache & $1(6.7)$ & $2(18.2)$ & 0.58 \\
\hline None & $9(60)$ & $5(45.5)$ & \\
\hline Coexisting hypoperfusion & $3(20.0)$ & $3(27.3)$ & \\
\hline None & $6(40.3)$ & $2(18.2)$ & \\
\hline Parietal & $2(13.4)$ & $2(18.2)$ & \\
\hline Parieto-temporal & $2(13.4)$ & $1(9.1)$ & 0.84 \\
\hline Parieto-occipital & $1(6.7)$ & $1(9.1)$ & \\
\hline Temporo-occipital & $1(6.7)$ & $2(18.2)$ & \\
\hline Temporal & $68.0 \pm 17.5$ & $70.0 \pm 20.9$ & \\
\hline K-MBI & & \\
\hline
\end{tabular}

Values are presented as mean \pm standard deviation or number (\%).

SPECT, single-photon emission computed tomography; TIA, transient ischemic attack; K-MBI, Korean version of the Modified Barthel Index. 
Table 3. Comparison of cognitive function in Moyamoya disease patients according to frontal-lobe perfusion

\begin{tabular}{|cccc|}
\hline & $\begin{array}{c}\text { With hypoperfusion } \\
\text { of the frontal lobe }(\mathbf{n = 1 5})\end{array}$ & $\begin{array}{c}\text { Without hypoperfusion } \\
\text { of the frontal lobe }(\mathbf{n}=\mathbf{1 1})\end{array}$ & p-value \\
\hline K-MMSE & $20.5 \pm 3.9$ & $24.0 \pm 5.1$ & 0.054 \\
\hline VCPT (s) & $0.7 \pm 0.2$ & $0.4 \pm 0.2$ & $0.001^{*}$ \\
\hline ACPT (s) & $0.8 \pm 0.1$ & $0.5 \pm 0.2$ & $<0.001^{*}$ \\
\hline FDST & $4.2 \pm 1.6$ & $6.1 \pm 2.3$ & $0.018^{*}$ \\
\hline BDST & $2.7 \pm 1.6$ & $4.5 \pm 2.0$ & $0.023^{*}$ \\
\hline FVST & $3.7 \pm 1.5$ & $4.2 \pm 2.5$ & 0.732 \\
\hline BVST & $3.1 \pm 1.5$ & $3.2 \pm 2.2$ & 0.937 \\
\hline ViLT & & & 0.932 \\
\hline First trial & $5.3 \pm 2.3$ & $5.4 \pm 3.4$ & 0.734 \\
\hline Fifth trial & $6.9 \pm 4.2$ & $7.0 \pm 4.2$ & 0.686 \\
\hline Retest & $7.2 \pm 5.4$ & $6.4 \pm 4.9$ & \\
\hline VeLT & & & $0.002^{*}$ \\
\hline First trial & $3.5 \pm 2.0$ & $6.3 \pm 2.2$ & 0.053 \\
\hline Fifth trial & $3.0 \pm 3.1$ & $5.3 \pm 2.5$ & $0.032^{*}$ \\
\hline Retest & $3.0 \pm 3.1$ & $5.6 \pm 2.5$ & $0.009^{*}$ \\
\hline TMT-A (s) & $124.7 \pm 73.6$ & $62.4 \pm 63.3$ & \\
\hline
\end{tabular}

Values are presented as mean \pm standard deviation.

K-MMSE, Korean version of the Mini-Mental Status Examination; VCPT, visual continuous performance test; ACPT, auditory continuous performance test; FDST, forward digit span test; BDST, backward digit span test; FVST, forward visual span test; BVST, backward visual span test; ViLT, visual learning test; VeLT, verbal learning test; TMT-A, trailmaking test-type $\mathrm{A}$.

* $\mathrm{p}<0.05$ by Mann-Whitney test.

(21.2) than in the PLN group (22.6), but this was not statistically significant $(\mathrm{p}=0.497)$ (Table 4$)$. Compared with the PLN group, brain SPECT of the PLH group showed lower scores in the backward visual span test, visual learning test, and trail-making test $(\mathrm{p}<0.05)$ (Table 5).

In the temporal and occipital lobes, there were no significant differences between the hypoperfusion and normal groups, with regard to baseline characteristics and CNT results.

\section{DISCUSSION}

In the current study, we analyzed the cognitive profiles of CNT in patients with MMD without focal cerebral lesions, and investigated the association with cerebral perfusion on brain SPECT. Our results are summarized as follows: (1) there was a high prevalence of cerebral hypoperfusion in MMD patients, even in the absence of lesions, and (2) the location of cerebral hypoperfusion showed significant correlation with the neurocognitive profile in each lobe.

In our study, only 3 of the 26 patients (11.5\%) showed normal perfusion cerebral SPECT. In patients with MMD, the chronic stenosis of brain vessels causes chronic hemodynamic stress, and induces formation of new collateral vessels [14]. Any increase in cerebral oxygen demand or a forced vasodilatation, such as acetazolamide challenge, paradoxically decreases the blood flow in the areas under threat, because the increased global cerebral perfusion due to vasodilatation significantly steals the blood flow from the affected cerebral cortex. Hayashi et al. [15] reported a high prevalence of cerebral hypoperfusion on cerebral angiography (81.1\%). In our study, the high prevalence of cerebral hypoperfusion ( $88.5 \%$ ) was consistent with these results.

On the basis of a previous study, almost all cognitive dysfunctions were caused by frontal, parietal, and temporal lobe lesions [7]; therefore, we categorized patients according to the hypoperfusion in the frontal, parietal, temporal, and occipital lobes. The frontal lobe is responsible for 
Table 4. Baseline characteristics of Moyamoya disease patients on parietal-lobe SPECT

\begin{tabular}{|c|c|c|c|}
\hline & $\begin{array}{c}\text { With hypoperfusion } \\
\text { of the parietal lobe }(n=10)\end{array}$ & $\begin{array}{l}\text { Without hypoperfusion } \\
\text { of the parietal lobe }(n=16)\end{array}$ & p-value \\
\hline Age (yr) & $39.3 \pm 13.0$ & $42.5 \pm 13.2$ & 0.32 \\
\hline Onset age (yr) & $38.5 \pm 12.7$ & $39.9 \pm 13.6$ & 0.74 \\
\hline Gender (male:female) & $1: 9$ & $6: 10$ & 0.32 \\
\hline Symptom duration (mo) & $11.0 \pm 22.4$ & $30.4 \pm 42.4$ & 0.23 \\
\hline Primary event & & & 0.83 \\
\hline TIA & $4(40.0)$ & $5(31.3)$ & \\
\hline Headache & $1(10.0)$ & $2(12.4)$ & \\
\hline None & $5(50.0)$ & $9(56.3)$ & \\
\hline Coexisting hypoperfusion & & & 0.45 \\
\hline None & $1(10.0)$ & $3(27.3)$ & \\
\hline Frontal & $6(67.0)$ & $2(18.2)$ & \\
\hline Fronto-temporal & $2(13.4)$ & $2(18.2)$ & \\
\hline Fronto-occipital & $1(6.7)$ & $1(9.1)$ & \\
\hline Temporo-occipital & $0(0.0)$ & $1(9.1)$ & \\
\hline Temporal & $1(6.7)$ & $2(18.2)$ & \\
\hline K-MBI & $67.2 \pm 17.7$ & $71.1 \pm 19.1$ & 0.74 \\
\hline
\end{tabular}

Values are presented as mean \pm standard deviation or number (\%).

SPECT, single-photon emission computed tomography; TIA, transient ischemic attack; K-MBI, Korean version of the Modified Barthel Index.

verbal short-term memory and attention, especially attention shift; therefore, patients with frontal lobe lesions showed abnormal findings on verbal learning test, word color test, and trail making test $[13,16]$. The parietal lobe is responsible for time-space orientation; therefore, patients with parietal lobe lesions showed abnormal findings on visual learning test $[12,17]$. In our study, MMD patients with chronic hypoperfusion in the frontal and parietal lobes showed similar patterns observed in previous studies. These results reflected that areas of chronic cerebral hemodynamic compromise devoid of focal cerebral lesions, were associated with specific cognitive dysfunctions. Another study showed that white matter was vulnerable to chronic ischemia [18]. The reported whitematter changes in chronic ischemia include demyelination, axonal loss, and gliosis [18-21]. A state of chronic hemodynamic compromise, arising from long-term hypoperfusion, may be the mechanism of cognitive dysfunction in this Moyamoya patient group. We observed that in the temporal and occipital lobe groups, there were no statistically significant findings between the hypoperfusion and normal groups. This may be due to the small number of patients in both groups.
The strength of our study was the use of brain SPECT with acetazolamide challenge that detects cerebral areas vulnerable to hypoperfusion very early [8]. In another study, patients who had cognitive impairment with normal cerebral MR perfusion showed abnormal brain SPECT with acetazolamide challenge test [22]. Thus, brain SPECT with acetazolamide challenge is capable of detecting cerebral hypoperfusion at the earliest. Mild cerebral hypoperfusion causes cognitive dysfunction prior to the appearance of focal lesion; hence, an early diagnosis and treatment of cerebral hypoperfusion is required.

Our study has several limitations. First, our patient sample size was small. Since cognitive function might be related with the lesion side [23], we could not analyze the lesion side due to limitation of sample size. Also, temporal lobe hypoperfusion might affect cognitive function; our study showed no significant differences between the temporal lobe hypoperfusion group and temporal lobe normal groups due to small sample size. Further studies with large sample size, especially for the temporal-lobe hypoperfusion, is required. Secondly, our sample was not sufficiently powered to detect potential differences in race, gender, or institution. Additional cases will be 
Table 5. Comparison of cognitive function in Moyamoya disease patients according to parietal-lobe perfusion

\begin{tabular}{|cccc|}
\hline & $\begin{array}{c}\text { With hypoperfusion } \\
\text { of the parietal lobe }(\mathbf{n}=\mathbf{1 0})\end{array}$ & $\begin{array}{c}\text { Without hypoperfusion } \\
\text { of the parietal lobe }(\mathbf{n}=\mathbf{1 6})\end{array}$ & p-value \\
\hline K-MMSE & $21.2 \pm 3.8$ & $22.6 \pm 5.1$ & 0.497 \\
\hline VCPT (s) & $0.7 \pm 0.2$ & $0.5 \pm 0.2$ & 0.364 \\
\hline ACPT (s) & $0.7 \pm 0.2$ & $0.6 \pm 0.2$ & 0.611 \\
\hline FDST & $3.8 \pm 1.9$ & $5.9 \pm 1.9$ & 0.152 \\
\hline BDST & $2.0 \pm 1.3$ & $4.5 \pm 1.8$ & 0.209 \\
\hline FVST & $3.3 \pm 1.6$ & $4.4 \pm 2.1$ & 0.231 \\
\hline BVST & $2.1 \pm 1.5$ & $3.7 \pm 1.8$ & $0.013^{*}$ \\
\hline ViLT & $3.8 \pm 2.6$ & & $0.015^{*}$ \\
\hline First trial & $4.2 \pm 4.1$ & $6.1 \pm 2.6$ & 0.083 \\
\hline Fifth trial & $5.4 \pm 6.4$ & $8.5 \pm 3.3$ & 0.334 \\
\hline Retest & & $7.9 \pm 3.6$ & \\
\hline VeLT & $3.7 \pm 1.7$ & & 0.572 \\
\hline First trial & $4.9 \pm 4.1$ & $5.3 \pm 2.7$ & 0.692 \\
\hline Fifth trial & $2.8 \pm 3.1$ & $8.1 \pm 3.2$ & 0.497 \\
\hline Retest & $154.5 \pm 72.5$ & $4.7 \pm 3.2$ & $0.048^{*}$ \\
\hline TMT-A (s) & $65.1 \pm 57.5$ & \\
\hline
\end{tabular}

Values are presented as mean \pm standard deviation.

K-MMSE, Korean version of the Mini-Mental Status Examination; VCPT, visual continuous performance test; ACPT, auditory continuous performance test; FDST, forward digit span test; BDST, backward digit span test; FVST, forward visual span test; BVST, backward visual span test; ViLT, visual learning test; VeLT, verbal learning test; TMT-A, trail making test-type $\mathrm{A}$.

* $\mathrm{p}<0.05$ by Mann-Whitney test.

needed to examine the effects of epidemiological and clinical variables on cognition.

Despite its limitations, this study was the first to correlate hypoperfusion territory and cognitive function in MMD patients using SPECT with acetazolamide challenge. Because early cognitive rehabilitation is important in cognitive impairment, we suggest starting early cognitive rehabilitation programs in MMD patients with hypoperfusion on SPECT without focal lesion $[24,25]$.

In conclusion, it is suggested that MMD patients had a high prevalence of cerebral hypoperfusion, and hypoperfusion areas without focal cerebral lesions was related to characteristics of neurocognitive dysfunction in MMD patients. Therefore, we suggest early evaluation of hypoperfusion by SPECT with acetazolamide challenge in MMD patients; early cognitive rehabilitation programs should be applied to MMD patients with hypoperfusion.

\section{CONFLICT OF INTEREST}

No potential conflict of interest relevant to this article was reported.

\section{REFERENCES}

1. Scott RM, Smith ER. Moyamoya disease and moyamoya syndrome. N Engl J Med 2009;360:1226-37.

2. Takeuchi K, Shimizu K. Hypogenesis of bilateral internal carotid artery. Shinkei 1957;9:37-43.

3. Cho HJ, Jung YH, Kim YD, Nam HS, Kim DS, Heo JH. The different infarct patterns between adulthood-onset and childhood-onset moyamoya disease. J Neurol Neurosurg Psychiatr 2011;82:38-40.

4. Ikezaki K, Matsushima T, Kuwabara Y, Suzuki SO, Nomura T, Fukui M. Cerebral circulation and oxygen metabolism in childhood moyamoya disease: a perioperative positron emission tomography study. J Neurosurg 1994;81:843-50. 
5. Abete P, Della-Morte D, Gargiulo G, Basile C, Langellotto A, Galizia G, et al. Cognitive impairment and cardiovascular diseases in the elderly: a heart-brain continuum hypothesis. Ageing Res Rev 2014;18:41-52.

6. Hynes SM, Fish J, Manly T. Intensive working memory training: a single case experimental design in a patient following hypoxic brain damage. Brain Inj 2014; 28:1766-75.

7. Kim JM, Lee SH, Roh JK. Changing ischaemic lesion patterns in adult moyamoya disease. J Neurol Neurosurg Psychiatr 2009;80:36-40.

8. Kuroda S, Houkin K. Moyamoya disease: current concepts and future perspectives. Lancet Neurol 2008;7: 1056-66.

9. Williams TS, Westmacott R, Dlamini N, Granite L, Dirks P, Askalan R, et al. Intellectual ability and executive function in pediatric moyamoya vasculopathy. Dev Med Child Neurol 2012;54:30-7.

10. Festa JR, Schwarz LR, Pliskin N, Cullum CM, Lacritz L, Charbel FT, et al. Neurocognitive dysfunction in adult moyamoya disease. J Neurol 2010;257:806-15.

11. Calviere L, Catalaa I, Marlats F, Januel AC, Lagarrigue J, Larrue V. Improvement in cognitive function and cerebral perfusion after bur hole surgery in an adult with moyamoya disease: case report. J Neurosurg 2011;115:347-9.

12. Jung HY, Park BK, Shin HS, Kang YK, Pyun SB, Paik NJ, et al. Development of the Korean Version of Modified Barthel Index (K-MBI): multi-center study for subjects with stroke. J Korean Acad Rehabil Med 2007;31:28397.

13. Kim YH, Shin SH, Park SH, Ko MH. Cognitive assessment for patient with brain injury by computerized neuropsychological test. J Korean Acad Rehabil Med 2001;25:209-16.

14. Weinberg DG, Arnaout OM, Rahme RJ, Aoun SG, Batjer HH, Bendok BR. Moyamoya disease: a review of histopathology, biochemistry, and genetics. Neurosurg Focus 2011;30:E20.

15. Hayashi K, Horie N, Izumo T, Nagata I. A nationwide survey on unilateral moyamoya disease in Japan. Clin Neurol Neurosurg 2014;124:1-5.

16. Milner B. Some cognitive effects of frontal-lobe lesions in man. Philos Trans R Soc Lond B Biol Sci 1982; 298:211-26.

17. Gitelman DR, Nobre AC, Parrish TB, LaBar KS, Kim YH, Meyer JR, et al. A large-scale distributed network for covert spatial attention: further anatomical delineation based on stringent behavioural and cognitive controls. Brain 1999;122 (Pt 6):1093-106.

18. Black S, Gao F, Bilbao J. Understanding white matter disease: imaging-pathological correlations in vascular cognitive impairment. Stroke 2009;40(3 Suppl):S48-52.

19. Kurumatani T, Kudo T, Ikura Y, Takeda M. White matter changes in the gerbil brain under chronic cerebral hypoperfusion. Stroke 1998;29:1058-62.

20. Riddle A, Dean J, Buser JR, Gong X, Maire J, Chen K, et al. Histopathological correlates of magnetic resonance imaging-defined chronic perinatal white matter injury. Ann Neurol 2011;70:493-507.

21. Shibata M, Ohtani R, Ihara M, Tomimoto H. White matter lesions and glial activation in a novel mouse model of chronic cerebral hypoperfusion. Stroke 2004; 35:2598-603.

22. Volkan-Salanci B, Lay Ergun E, Genc Sel C, Yalnizoglu $D$, Turanli G. The role of brain perfusion SPECT in moyamoya disease. Rev Esp Med Nucl Imagen Mol 2012;31:216-8.

23. Kim SK, Oh JK, Lee EJ. Neurobehavioral cognitive status examination in stroke patients. J Korean Acad Rehabil Med 1997;21:259-63.

24. Adamski N, Adler M, Opwis K, Penner IK. A pilot study on the benefit of cognitive rehabilitation in Parkinson's disease. Ther Adv Neurol Disord 2016;9:15364.

25. Feng H, Li G, Xu C, Ju C, Qiu X. Training rehabilitation as an effective treatment for patients with vascular cognitive impairment with no dementia. Rehabil Nurs 2016. 